\title{
Desempenho ocupacional, qualidade de vida e adesão ao tratamento de pacientes com epilepsia
}

\section{Occupational performance, quality of life and adherence to treatment of patients with epilepsy}

\author{
Cristiane Carnaval Gritti ${ }^{1}$, Vânia Uemura Paulino² ${ }^{2}$ Lúcia Helena Neves Marques³, \\ Lilian Castiglioni ${ }^{4}$, Maysa Alahmar Bianchin ${ }^{5}$
}

http://dx.doi.org/10.11606/issn.2238-6149.v26i1p93-101

Gritti CC, Paulino VU, Marques LHN, Castiglioni L, Bianchin MA. Desempenho ocupacional, qualidade de vida e adesão ao tratamento de pacientes com epilepsia. Rev Ter Ocup Univ São Paulo. 2015 jan./abr.;26(1):93-101.

RESUMO. Os objetivos do estudo foram avaliar o desempenho ocupacional, qualidade de vida e adesão ao tratamento medicamentoso de pacientes com epilepsia de difícil controle. Trata-se de um estudo transversal e quantitativo com 30 pacientes em acompanhamento no ambulatório de neurologia em epilepsia de difícil controle da no ambulatório do Hospital de Base de São José do Rio Preto. Os instrumentos utilizados foram: ficha de Identificação, Teste de Morisky, Qualityof Life in Epilepsy Inventory - 31 e Medida Canadense de Desempenho Ocupacional. Os resultados apontaram que: $70 \%$ dos participantes obtiveram média adesão ao tratamento medicamentoso; em relação à qualidade de vida os piores resultados foram a preocupação com as crises e os efeitos adversos das drogas antiepilépticas. No Desempenho Ocupacional, as principais dificuldades levantadas constataram prevalência no funcionamento na comunidade, trabalho e socialização. O estudo possibilitou identificar o comportamento do paciente em relação ao uso da medicação, mensurar a qualidade de vida no contexto vivenciado pela doença e identificar as áreas variadas de desempenho ocupacionais prejudicadas.

DESCRITORES: Epilepsia; Qualidade de vida; Desempenho ocupacional; Adesão à medicação.
Gritti CC, Paulino VU, Marques LHN, Castiglioni L, Bianchin MA. Occupational performance, quality of life and adherence to treatment of patients with epilepsy.Rev Ter Ocup Univ São Paulo. 2015 jan./abr.;26(1):93-101.

ABSTRACT. To assess occupational performance, quality of life and adherence to drug therapy of patients with hard-tocontrol epilepsy. This is a quantitative, cross-sectional study. They were selected thirty patients diagnosed with difficultto-control epilepsy who were being treated in the outpatient Clinic of Hospital de Base in São José do Rio Preto. Subjects were evaluated using the Morisky test, the Quality of Life in Epilepsy-31 Questionnaire, and the Canadian Occupational Performance Measure. The Morisky test identified that $70 \%$ of patients had medium adherence to drug treatment, in relation to quality of life the worst results were compared to seizure worry and adverse effects of antiepileptic drugs. The main difficulties reported in the Canadian Occupational Performance Measure were: functioning in the community, work and socialization. The instruments used by occupational therapists allow to evaluate and and subsequently possible for the therapeutic targeting key areas of occupational performance compromised.

KEYWORDS: Epilepsy; Quality of life; Occupational performance; Medication adherence.

Resultados parciais deste estudo foram apresentados em sessão de pôster eletrônico durante o XIII Congresso Brasileiro - Terapia Ocupacional e Políticas Públicas: diretrizes, compromissos e ações, realizados no em Florianópolis em outubro de 2013 e no X Congresso Anual de Iniciação Científica da FAMERP em São José do Rio Preto em 2013.

1.Terapeuta Ocupacional. Residente de Terapia Ocupacional em Reabilitação Física. Faculdade de Medicina de São José do Rio Preto

(FAMERP), São José do Rio Preto. Email: cris.gritti@hotmail.com.

2.Terapeuta Ocupacional. Especialista em Terapia Ocupacional: uma visão dinâmica em Neurologia.

3.Doutora em Ciências da Saúde pela Faculdade de Medicina de São José do Rio Preto - FAMERP e Neurologista do Centro de Cirurgia

de Epilepsia do Hospital de Base e da Faculdade de Medicina de São José do Rio Preto.

4.Doutora em Genética pela UNESP - Campus São José do Rio Preto.

5.Terapeuta Ocupacional - Prof ${ }^{a}$ Adjunta do Departamento de Ciências Neurológicas da Faculdade de Medicina de São José do Rio

Preto (FAMERP), São José do Rio Preto. Email: maysa@famerp.br.

Endereço para correspondência: Maysa A. Bianchin - Faculdade de Medicina de São José do Rio Preto, Departamento de Ciências Neurológicas. Av. Brigadeiro Faria Lima, 5416 - Vila São Pedro. CEP: 15090-000. São José do Rio Preto, SP, Brasil. 


\section{INTRODUÇÃO}

$\mathrm{A}$ tualmente, as doenças crônicas estão entre os principais e mais frequentes problemas de saúde. Destaca-se a epilepsia devido suas características clínicas peculiares, como cronicidade, crises recorrentes, geralmente não provocadas e imprevisíveis. A crise epiléptica é caracterizada como disfunção fisiológica temporária do cérebro devido a uma descarga anormal excessiva e transitória de neurônios corticais ${ }^{1,2,3,4}$

O diagnóstico de epilepsia é feito quando ocorrem, pelo menos, duas ou mais crises em um período de doze meses, na ausência de doença tóxico-metabólica ou estado febril. Em pacientes com epilepsia, a forma de tratamento medicamentoso com uso de drogas antiepilépticas tem o objetivo de eliminar ou reduzir as crises. Desta forma, o tratamento contribuirá para a melhoria da qualidade de vida, além de reduzir o impacto da doença para o indivíduo nos contextos social, de lazer, de trabalho e escolar.

Alguns fatores modificáveis podem dificultar o controle das crises, como estresse, privação de sono e baixa adesão ao tratamento ${ }^{5,6,7}$. A adesão ao tratamento medicamentoso é considerada como "a utilização dos medicamentos prescritos ou outros procedimentos em pelo menos $80 \%$ de seu total, observando horários, doses e tempo de tratamento" 8.9 .

Quando não há controle satisfatório das crises com o uso de monoterapia, associam-se mais medicamentos. Caso não haja o controle das crises pela politerapia, denomina-se que este paciente é de difícil controle. Conceitualmente a epilepsia de difícil controle se configura quando o paciente não alcançou o controle aceitável das crises mesmo utilizando as drogas antiepilépticas eficazes, com níveis e doses máximas. ${ }^{10}$

No Brasil, estima-se que existam três milhões de pessoas com epilepsia, sendo que a cada novo dia, somamse a este quadro cerca de trezentos novos casos $^{8}$. Além disto, a epilepsia, pela sua cronicidade e apresentação clínica é uma das doenças que mais afeta o comportamento e a qualidade de vida, sendo, portanto, um grande desafio para os profissionais de saúde ${ }^{11}$. A Qualidade de Vida (QV) é um conceito amplo que engloba a saúde física, o estado psicológico, nível de independência, as relações sociais, as crenças pessoais, a relação com o meio ambiente e desempenho ocupacional ${ }^{12,13,14}$.

Desempenho é "descreve, como qualificador, o que os indivíduos fazem no seu ambiente habitual incluindo assim o aspecto do envolvimento nas situações da vida." ${ }^{15}$. O termo ocupação envolve todo o esforço produtivo humano, desempenhado e vivido; em contextos de trabalho, lazer, cuidado pessoal e outros. É identificado na rotina cotidiana das atividades nas quais o individuo se envolve, preenchendo seu tempo e trazendo significado para sua vida ${ }^{16,17}$.

Assim, desempenho ocupacional caracteriza-se como a habilidade do indivíduo realizar suas atividades cotidianas, sendo influenciado por papéis ocupacionais, componentes de desempenho e ambiente. $\mathrm{O}$ desempenho ocupacional é independe da idade, gênero e incapacidade e envolve três áreas integradas: o autocuidado, a produtividade e o lazer; conforme as exigências do ambiente externo e do meio interno da pessoa ${ }^{18,19}$.

$\mathrm{O}$ atendimento dos pacientes com epilepsia é feito pela equipe multiprofissional. Como componente da equipe o terapeuta ocupacional está capacitado para otimizar o desempenho ocupacional dos indivíduos, para possibilitar a realização suas atividades cotidianas, permitindo maior qualidade de vida ${ }^{20,21,22}$.

Diante do exposto, este estudo teve como objetivo a avaliação do desempenho ocupacional, qualidade de vida e adesão ao tratamento medicamentoso de pacientes com diagnóstico de epilepsia de difícil controle.

\section{MÉTODO}

O estudo foi realizado, transversal e quantitativo, foi realizado no período de maio de 2013 a setembro de 2013, no ambulatório de neurologia em epilepsia de difícil controle do Hospital de Base de São José do Rio Preto. O projeto foi aprovado pelo Comitê de Ética em Pesquisa com seres humanos da FAMERP, pelo protocolo $n^{\circ} 011702 / 2012$.

Foram selecionados 30 pacientes em acompanhamento ambulatorial há mais de um ano por quadro de epilepsia de difícil controle. Antes de iniciar qualquer procedimento o Termo de Consentimento Livre e Esclarecido foi assinado por todos os participantes.

Pacientes que apresentaram, no período do estudo, doenças psiquiátricas ou neurológicas progressivas, que comprometessem a compreensão dos testes ou aqueles que não aceitaram participar da pesquisa, foram excluídos da pesquisa.

\section{Descrição dos instrumentos}

Todos os instrumentos foram aplicados pelo 
mesmo terapeuta ocupacional na seguinte ordem: ficha de Identificação, Teste de Morisky, Qualityof Life in Epilepsy Inventory - 31 (QOLIE-31) e Medida Canadense de Desempenho Ocupacional (COPM).

Pela ficha de identificação foram coletados os dados demográficos (nome, idade, gênero, escolaridade, estado civil, atividade laboral, número de filhos, religião e atividade de lazer), além dos registros clínicos como: diagnóstico, início das crises, se realizou ou não procedimento cirúrgico para epilepsia e a quantidade de medicamentos antiepilépticos utilizadas.

O Teste de Morisky consiste em quatro perguntas que avaliam o comportamento do paciente em relação ao uso da medicação, classificando sua adesão terapêutica. As respostas são dicotômicas ( $\operatorname{sim}$ ou não) e para a classificação de baixa, média ou alta adesão é feito a soma de respostas positivas e de respostas negativas ${ }^{23,24}$.

O Qualityof Life in Epilepsy Inventory - 31, mensura a qualidade de vida do paciente com epilepsia. Possui 31 questões que avaliam: preocupação com as crises epilépticas, a qualidade de vida global, sociabilidade, energia/fadiga, aspectos emocionais, aspectos cognitivos e efeitos adversos das drogas antiepilépticas (DAEs). Escore total de cada um destes domínios varia de 0 à 100 , no qual 0 representa nenhuma qualidade de vida e 100 representa melhor qualidade de vida. A qualidade de vida é diretamente proporcional à maior pontuação do escore. Além disto, a questão 31 deste instrumento é analisada separadamente calculando apenas a média e o desvio padrão, no qual o participante deveria responder um valor de 0 (pior saúde possível) a 100 (melhor saúde possível) de como este se sentia em relação a sua saúde considerando a epilepsia ${ }^{25,26}$.

A Medida Canadense de Desempenho Ocupacional - Canadian Measure of Occupational Performance COPM utilizada por terapeutas ocupacionais, foi criada para medir mudanças na autopercepção do desempenho ocupacional de indivíduos com diferentes incapacidades e em qualquer estágio de desenvolvimento. Engloba as áreas de autocuidado, mobilidade funcional e o funcionamento na comunidade. $\mathrm{Na}$ área de produtividade são considerados aspectos do trabalho, manejo nas tarefas domésticas, na escola e no brincar. A área de lazer inclui recreação tranquila, recreação ativa e socialização. Trata-se de uma entrevista semi-estruturada e individualizada, na qual o indivíduo pontua as atividades cotidianas que são importantes e que ele tem dificuldade. Neste estudo, o instrumento foi utilizado com o objetivo de identificar as áreas de desempenho ocupacional prejudicadas, de forma que foram apenas elencadas pelo indivíduo as atividades cotidianas consideradas importantes e nas quais cada indivíduo apresentava dificuldades ${ }^{18,27,28}$.

\section{Análise estatística dos dados}

Foi realizada a análise descritiva dos dados obtidos, a verificação da normalidade dos dados pelo Teste de Kolmogorov-Smirnov (KS) e foram aplicados testes estatísticos paramétricos, utilizados para amostras que apresentam valores sem distribuição normal. Para as comparações envolvendo dois grupos independentes foi aplicado o Teste $\mathrm{T}$ de Student. O nível de significância adotado foi de $p<0,05$. A análise estatística dos dados obtidos foi realizada com o auxílio do software GraphPadInstat 3.0.

\section{RESULTADOS}

Os dados demográficos e os dados clínicos obtidos pela ficha de identificação são apresentados na Tabela 1. A idade dos 30 participantes variou entre 20 e 71 anos ( $\dot{M}$ $45 \pm 11$ ), sendo o gênero feminino o de maior prevalência $(67 \%)$.

A Tabela 2 apresenta os resultados do Teste de Morisky, no qual se observa que $70 \%$ dos participantes obtiveram média adesão ao tratamento medicamentoso.

A média e o desvio padrão dos domínios avaliados pela QOLIE-31, além do valor total e da questão 31 deste instrumento são apresentados na Tabela 3, onde a preocupação com as crises apresentou pior resultado e os efeitos adversos das DAEs o melhor.

Por meio da COPM, pode-se constatar que no autocuidado $70 \%$ dos participantes relataram dificuldade no funcionamento na comunidade, mas na categoria produtividade $67 \%$ referiram maior dificuldade no trabalho remunerado/não remunerado e no cuidar de tarefas domésticas não referiram dificuldade (74\%). Já no lazer, a maioria não apresentou dificuldades, tendo a recreação calma/tranquila obtido a maior prevalência $(80 \%)$ dos três itens avaliados.

O teste $T$ de Student permitiu comparar os valores totais da QOLIE-31 entre os indivíduos que praticavam ou não atividades de lazer, determinando que os participantes que não as realizavam apresentavam o valor total da QOLIE significativamente menor do que aqueles que as praticavam $(\mathrm{P}$ valor $=0,0002)$, (Figura 1$)$. 
Tabela 1 - Dados de demográficos e clínicos

\begin{tabular}{|c|c|}
\hline Dados & Frequência (\%) \\
\hline \multicolumn{2}{|l|}{ Gênero } \\
\hline Feminino & $20(67 \%)$ \\
\hline Masculino & $10(33 \%)$ \\
\hline \multicolumn{2}{|l|}{ Escolaridade } \\
\hline Ensino fund. incompleto & $15(50 \%)$ \\
\hline Ensino fund. completo & $6(20 \%)$ \\
\hline Ensino médio incompleto & $2(7 \%)$ \\
\hline Ensino médio completo & $5(16 \%)$ \\
\hline Ensino superior completo & $2(7 \%)$ \\
\hline \multicolumn{2}{|l|}{ Estado Civil } \\
\hline Casado & $16(54 \%)$ \\
\hline Solteiro & $5(17 \%)$ \\
\hline Divorciado & $4(13 \%)$ \\
\hline Amasiado & $4(13 \%)$ \\
\hline Viúvo & $1(3 \%)$ \\
\hline \multicolumn{2}{|l|}{ Atividade laboral } \\
\hline Desempregados & $15(50 \%)$ \\
\hline Atividade remunerada & $7(23 \%)$ \\
\hline Aposentados ou afastados & $6(20 \%)$ \\
\hline Atividade não remunerada & $2(7 \%)$ \\
\hline \multicolumn{2}{|l|}{ Número de Filhos } \\
\hline 0 & $12(40 \%)$ \\
\hline 1 & $5(17 \%)$ \\
\hline 2 & $10(33 \%)$ \\
\hline 3 & $1(3 \%)$ \\
\hline 4 ou mais & $2(7 \%)$ \\
\hline \multicolumn{2}{|l|}{ Religião } \\
\hline Católico & $23(77 \%)$ \\
\hline Evangélico & $4(13 \%)$ \\
\hline Espírita & $2(7 \%)$ \\
\hline Nenhuma & $1(3 \%)$ \\
\hline \multicolumn{2}{|l|}{ Atividades de Lazer } \\
\hline Sim & $22(73 \%)$ \\
\hline Não & $8(27 \%)$ \\
\hline \multicolumn{2}{|l|}{ Diagnóstico } \\
\hline Epilepsia de lobo temporal & $24(80 \%)$ \\
\hline Epilepsia de lobo frontal & $2(7 \%)$ \\
\hline Epilepsia mioclônica Juvenil & $2(7 \%)$ \\
\hline Epilepsia pós meningite fronto temporal & $1(3 \%)$ \\
\hline Epilepsia Pós TCE de lobo temporal & $1(3 \%)$ \\
\hline \multicolumn{2}{|l|}{ Início das crises } \\
\hline Infância & $19(63 \%)$ \\
\hline Adolescência & $4(13 \%)$ \\
\hline Adulto & $7(23 \%)$ \\
\hline \multicolumn{2}{|l|}{ Procedimento cirúrgico de epilepsia } \\
\hline Não & $21(70 \%)$ \\
\hline Sim & $9(30 \%)$ \\
\hline \multicolumn{2}{|l|}{ Tratamento com drogas antiepilépticas } \\
\hline Monoterapia & $7(23 \%)$ \\
\hline Politerapia & $23(77 \%)$ \\
\hline
\end{tabular}

*TCE:traumatismo crânio-encefálico 
Tabela 2 - Teste de Morisky - adesão ao tratamento medicamentoso

\begin{tabular}{ccc}
\hline Morisky & Número de Pacientes & Porcentagem \\
\hline Baixa adesão & 2 & $7 \%$ \\
Média adesão & 21 & $70 \%$ \\
Alta adesão & 7 & $23 \%$ \\
\hline
\end{tabular}

Tabela 3 - Resultado de cada domínio, resultado total e questão 31 da QOLIE-31

\begin{tabular}{lll}
\hline Domínios & Média & Desvio Padrão \\
\hline Preocupação com as crises & 28,31 & 28,61 \\
Qualidade de vida global & 43,41 & 24,92 \\
Sociabilidade & 46,23 & 26,38 \\
Energia/Fadiga & 51,00 & 27,24 \\
Aspectos emocionais & 53,90 & 25,37 \\
Aspectos cognitivos & 57,88 & 25,46 \\
Efeitos adversos das DAEs & 66,70 & 30,80 \\
Total & 49,69 & 19,14 \\
Questão 31 & 73,50 & 21,56 \\
\hline
\end{tabular}

*DAEs: drogas antiepilépticas

Tabela 4 - Medida Canadense de Desempenho Ocupacional - COPM

\begin{tabular}{|c|c|c|c|c|}
\hline COPM & $\begin{array}{l}\text { Sim, apresentam } \\
\text { dificuldades }\end{array}$ & $\%$ & $\begin{array}{l}\text { Não apresentam } \\
\text { dificuldades }\end{array}$ & $\%$ \\
\hline \multicolumn{5}{|l|}{ Autocuidado } \\
\hline Cuidados Pessoais & 7 & $23 \%$ & 23 & $77 \%$ \\
\hline Mobilidade Funcional & 8 & $27 \%$ & 22 & $73 \%$ \\
\hline Funcionamento na comunidade & 21 & $70 \%$ & 9 & $30 \%$ \\
\hline \multicolumn{5}{|l|}{ Produtividade } \\
\hline Trabalho remunerado/não remunerado & 20 & $67 \%$ & 10 & $33 \%$ \\
\hline Cuidar de tarefas de casa & 8 & $26 \%$ & 22 & $74 \%$ \\
\hline Brincar/Escola & - & - & - & - \\
\hline \multicolumn{5}{|l|}{ Lazer } \\
\hline Recreação calma/tranquila & 6 & $20 \%$ & 24 & $80 \%$ \\
\hline Recreação ativa & 9 & $30 \%$ & 11 & $70 \%$ \\
\hline Socialização & 10 & $33 \%$ & 20 & $67 \%$ \\
\hline
\end{tabular}

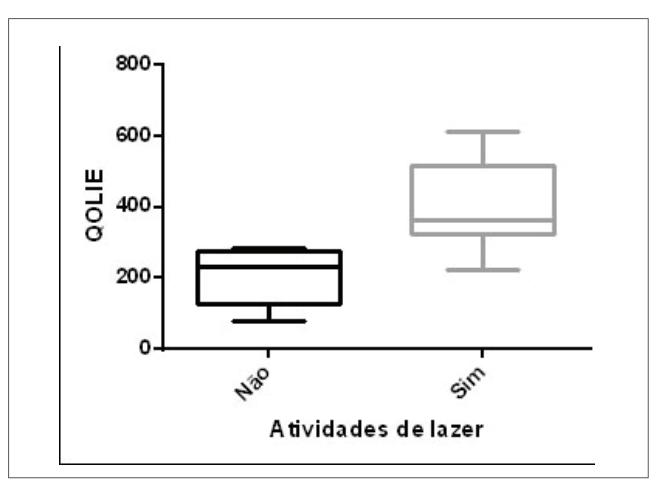

Figura 1 - Correlação do valor total da QOLIE-31 entre os indivíduos que praticavam ou não atividade de lazer (Teste $\mathrm{T}$ de Student)

\section{DISCUSSÃO}

Este estudo buscou avaliar o desempenho ocupacional, qualidade de vida e adesão ao tratamento medicamentoso de pacientes com epilepsia de difícil controle e dar visibilidade aos instrumentos utilizados na prática clínica da terapia ocupacional.

$\mathrm{O}$ perfil demográfico dos participantes mostrou maior prevalência do gênero feminino (67\%), sendo que em alguns estudos apresentam que a frequência deste gênero é de $53,5 \%$ a $62,1 \%$ das pessoas adultas com epilepsia ${ }^{29,30}$. Entretanto, outros estudos observaram que há ligeiramente maior incidência do gênero masculino ${ }^{31,32,33}$.

Com relação às características clínicas da doença, 
os resultados mostraram que a maioria (80\%) apresentou o diagnóstico de Epilepsia de Lobo Temporal. Este dado pode estar relacionado ao fato de que a epilepsia do lobo temporal é o diagnóstico mais comum de epilepsia de difícil controle ${ }^{34}$.

Quanto ao tratamento medicamentoso, constatouse maior prevalência $(77 \%)$ de utilização da politerapia medicamentosa, confirmando que pacientes com diagnóstico de epilepsia de difícil controle geralmente necessitam de politerapia medicamentosa ${ }^{35}$. Nos pacientes em que não há controle das crises com a monoterapia, utiliza-se a combinação de duas ou mais DAEs ${ }^{36}$.

No Teste de Morisky, 70\% dos participantes da pesquisa tiveram média adesão ao tratamento medicamentoso, resultado concordante com outro estudo, no qual o teste foi aplicado sendo constatado que $60,5 \%$ dos pacientes obtiveram Média Adesão ao Tratamento Medicamentoso ${ }^{35}$. Pacientes que não utilizam corretamente os medicamentos referem dificuldade para controlar as crises comparados com aqueles que fazem o uso conforme orientado. A não adesão adequada dos pacientes com epilepsia podem estar relacionadas a alguns fatores, como relacionadas ao próprio paciente (demografia, situação socioeconômica, suas percepções e crenças relacionadas a epilepsia), a doença (quanto ao número de crises e sua gravidade), a medicação (número de medicamentos, de comprimidos e doses; seus efeitos colaterais e orientações especiais para o uso), a relação entre paciente-médico, quanto ao paciente esquecer de tomá-los, o alto custo e outras dificuldades para aquisição destes medicamentos, complexidade terapêutica ${ }^{37,38.39,40}$.

Notou-se, utilizando o instrumento QOLIE-31, que maior média encontrada foi no domínio: efeitos adversos das DAEs e os domínios que obtiveram menor média foi preocupação com as crises, seguido pela qualidade de vida global e sociabilidade, dado este confirmado pela literatura $^{41}$. Em outro estudo, que avaliou 34 sujeitos com epilepsia, o pior índice encontrado foi "preocupação com as crises", porém o segundo menor índice foi do "efeito dos medicamentos" ${ }^{42}$. Vale ressaltar, que nos indivíduos com epilepsia a qualidade de vida é diretamente determinada pela forma com a qual este vive com a condição crônica, ou seja, não é determinada pela doença em si ${ }^{43,44,45}$. Sendo assim podemos observar nestes diferentes estudos que a preocupação com as crises é um dos principais fatores que a pessoa com epilepsia refere que esta condição crônica afeta na sua vida.

Com relação ao Desempenho Ocupacional, avaliadas pela COPM, pode-se constatar que no autocuidado os participantes relataram dificuldades principalmente no funcionamento na comunidade vinculado a: dirigir carro, usar transporte público, manipular dinheiro, dar troco e pagar contas. No item produtividade referiram mais dificuldade trabalho remunerado/não remunerado, como procurar trabalho, preparar-se para a entrevista, selecionar e gerenciar responsabilidade. Dados compatíveis com os estudos de Nickel et al. de $1999^{42}$ e de $2001^{46}$. E por fim no lazer, referiram dificuldades na socialização (visitar família/amigos, falar ao telefone, frequentar festas e atividades). Consideramos que isto pode ser devido a alguns fatores restringirem o desempenho e as atividades de vida diária, como o própria condição crônica ou até a falta de conhecimento sobre epilepsia pela comunidade c $^{47,48,49}$.

Podemos ainda justificar que algumas dificuldades nas atividades do desempenho ocupacional podem ser restringidas pela própria preocupação dos pacientes em se sentirem envergonhados ou terem problemas sociais devido às crises.

Observamos, com os dados obtidos e correlacionados na COPM, que 74\% não apresentaram dificuldades nas atividades de casa, porém $67 \%$ referiram dificuldades no trabalho remunerado/não remunerado, provavelmente indicando que no contexto laboral há mais situações estressantes e maiores cobranças do que no ambiente domiciliar. Além disto, as dificuldades para a obtenção e manutenção do trabalho podem estar relacionadas ao estigma social a respeito da epilepsia e ao nível de escolaridade e a dificuldade em frequentar escolas e cursos. Sendo assim, as pessoas com epilepsia apresentam maior dificuldade de conseguir um emprego formal ${ }^{47,50}$.

No presente estudo foi determinado utilizando o Teste $\mathrm{T}$, valor altamente significativo $(\mathrm{P}$ valor $=0,0002)$ na comparação entre os valores totais da QOLIE-31 e os indivíduos que praticavam ou não atividades de lazer, destacando a importância das atividades de lazer para melhor qualidade de vida. Assim, podemos considerar que as atividades prazerosas como as de lazer estão diretamente relacionadas a uma melhor qualidade de vida, sendo de grande importância às recreações e a socialização. Destacase a importância da (re)inserção social das pessoas com epilepsia, para que desempenhem suas atividades de lazer satisfatoriamente, bem como as atividades produtivas e de autocuidado.

Este estudo forneceu informações sobre a média adesão ao tratamento medicamentoso, as áreas mais afetadas da qualidade de vida e as limitações do desempenho ocupacional vivenciadas pelos pacientes com epilepsia de difícil controle. Diante dos resultados apresentados tornouse mais evidente a importância da participação efetiva da equipe interdisciplinar e o papel do terapeuta ocupacional 
que poderá direcionar sua intervenção nas principais áreas de desempenho ocupacional comprometidas. Além disso, ainda como trabalho terapêutico ocupacional, intervir através de

\section{REFERÊNCIAS}

1. Bragatti JA, Torres CM, Londero RG, Martin $\mathrm{KC}$, Souza AC, Hidalgo MP, Chaves ML, Bianchin MM. Prevalence of psychiatric comorbidities in temporal lobe epilepsy in a Southern Brazilian population.Arq Neuro-Psiquiatr. 2011;69(2):159-65. doi: 10.1590/S0004282X2011000200003.

2. Banerjee PN, Filippi D, Hauser WA.The descriptive epidemiology of epilepsy - a review. Epilepsy Res. 2009;85(1):31-45. doi: 10.1016/j.eplepsyres.2009.03.003.

3. Germaine-Smith CS, Liu M, Quan H, Wiebe S, Jette N. Development of an epilepsy-specific risk adjustment comorbidity index. Epilepsia. 2011;52(12):2161-7. doi: 10.1111/j.1528-1167.2011.03292.x.

4. Guilhoto LMFF. Revisão terminológica e conceitual para organização de crises e epilepsias: relato da comissão da ILAE de classificação e terminologia, 2005-2009. Novos paradigmas? J Epilepsy Clin Neurophsiol. 2011;17(3):1005. doi: 10.1590/S1676-26492011000300005

5. Faught E, Richman J, Martin R, Funkhouser E, Foushee R, Kratt P, Kim Y, Clements K, Cohen N, Adoboe D, Knowlton $\mathrm{R}$, Pisu M. Incidence and prevalence of epilepsy among older US Medicare beneficiaries. Neurology. 2012;78(7):448-53. doi: 10.1212/WNL.0b013e3182477edc.

6. Fountain NB, Van Ness PC, Swain-Eng R, Tonn S, Bever CT Jr. Quality improvement in neurology: AAN epilepsy quality measures: Report of the Quality Measurement and Reporting Subcommittee of the American Academy of Neurology. Neurology. 2011;76(1):94-9. doi: 10.1212/01 wnl.0000437305.37850.f9.

7. Handoloko KB, Zwart-van Rijkom JEF, Visee HF, Hermes WAJJ, Hekester YA, Egberts TCG. Drug treatment-related factors of inadequate seizure control. Epilepsy Behav. 2008;13:545-8. doi: 10.1016/j.yebeh.2008.04.007.

8. Gimenes HT, Zanetti ML, Haas VJ. Fatores relacionados à adesão do paciente diabético à terapêutica medicamentosa. Rev Latino-Am Enferm. 2009;17(1):46-51. doi: 10.1590/ S0104-11692009000100008.

9. Blaschke TF, Osterberg L, Vrijens B, Urquhart J. Adherence to medications: insights arising from studies on the unreliable link between prescribed and actual drug dosing histories annual review of pharmacology and toxicology. Pharmacol Toxicol. 2012;52:275-301. doi: 10.1146/annurevpharmtox-011711-113247. palestras educativas, no intuito de diminuir diversas dúvidas, facilitar e conscientizar a adesão medicamentosa e melhorar a percepção em relação à doença.

10. Garzon E. Epilepsia refratária: conceito e contribuição das novas drogas antiepilépticas e de outras modalidades terapêuticas. Rev Neurociências. 2002;10(2):66-82. Disponível em: http://www.revistaneurociencias.com.br/ edicoes/2002/RN\%2010\%2002/Pages $\% 20$ from $\% 20 \mathrm{RN} \% 20$ $10 \% 2002-3 . p d f$

11. Fernandes PT, Min LS, Ling LH, Correa L, Barros NF, Li LM. Grupos de Interação Social (GIS): estratégia de empowerment para pessoas com epilepsia. J Epilepsy Clin Neurophsiol. 2011;17(2):70-4. doi: 10.1590/S167626492011000200007.

12. Versão em português dos instrumentos de avaliação de qualidade de vida (WHOQOL) 1998 (Internet). Rio Grande do Sul: Universidade Federal do Rio Grande do Sul; 29 jul. 2007 [citado 24 abr. 2012]. Disponível em: www.ufrgs.br/ psiq/whoqol84.html.

13. Leal CMS, Reavaliar o conceito de qualidade de vida. Obtido em 10 de fevereiro de 2012. Disponível em: http:// www.porto.ucp.pt/lusobrasileiro/actas/Carla\%20Leal.pdf.

14. Theofilou P. Quality of life: definition and measurement. Europe's J Psychol. 2013;9(1):150-62. doi: 10.5964/ejop. v9i1.337.

15. Organização Mundial da Saúde - Classificação Internacional de Funcionalidade, Incapacidade e Saúde, CIF. [Centro Colaborador da Organização Mundial da Saúde para a Família de Classificações Internacionais, org.; coordenação da tradução Cassia Maria Buchalla]. São Paulo: EDUSP; 2003.

16. AOTA - The American Occupational Therapy Association. Occupational therapy practice framework: domain and process. Am J Occup Ther. 2002;56:609-39.

17. Lima EMFA, Okuma DG, Pastor MN.Atividade, ação, fazer e ocupação: a discussão dos termos na Terapia Ocupacional brasileira. Cad Ter Ocup UFSCar. 2013; 21(2):243-54. doi: $10.4322 \% 2$ Fcto. 2013.026

18. Zanni KP, Bianchin MA, Marques LHN. Qualidade de vida e desempenho ocupacional de pacientes submetidos à cirurgia de epilepsia. J Epilepsy Clin Neurophysiol. 2009;15(3):114-7. doi: 10.1590/S167626492009000300004.

19. Chapparo C, Ranka J. The occupational performance model (Australia): A description of constructs and structure. Sidney, Australia: The Universith of Sidney; 1997. 
20. Engel J Jr. Progress in the field of epilepsy. Curr Opin Neurol. 2013;26:160-2. doi: 10.1097/WCO.0b013e32835ee5a3.

21. Wedlund EW, Nilsson L, Tomson T, Erdner A. What is important in rehabilitation for persons with epilepsy? Experiences from focus group interviews with patients and staff. Epilepsy Behav. 2013;28(3):347-53. doi: 10.1016/j. yebeh.2013.05.022.

22. Wedlund WE, Nilsson L, Erdner A, Tomson T. Long term follow-up after comprehensive rehabilitation of persons with epilepsy, with emphasis on participation in employment or education. Epilepsy Behav. 2012;25(2):219-23. doi: 10.1016/j.yebeh.2012.06.029.

23. Ben AJ, Neumann CR, Mengue SS. Teste de Morisky-Green e Brief Medication Questionnaire para avaliar adesão a medicamentos. Rev Saúde Pública. 2012;46(2):279-89. doi: 10.1590/S0034-89102012005000013.

24. Morisky DE, Green LW, Levine DM. Concurrent and predictive validity of a self-reported measure of medication adherence. Med Care. 1986;24(1):67-74. doi: 10.1097/00005650-198601000-00007.

25. Da Silva TI, Ciconelli RM, Alonso NB, Azevedo AM, Westphal-Guitti AC, Pascalicchio TF, et al. Validity and reliability of the Portuguese version of the quality of life in epilepsy inventory (QOLIE-31) for Brazil. Epilepsy Behav. 2007;10(2):234-41. doi: 10.1590/S167626492008000100009.

26. Da Silva TI, Marques CM, Alonso NB, Azevedo AM, Westphal-Guitti AC, Caboclo LOSF, Sakamoto AC, Yacubian EMT. Tradução e Adaptação Cultural do Quality of Life in Epilepsy (QOLIE-31). J Epilepsy Clin Neurophsiol. 2006;12(2):107-10. doi: 10.1590/S167626492006000300011.

27. Chen YH, Rodger S, Polatajko H. Experiences with the COPM and client-centred practice in adult neurorehabilitation in Taiwan. 2002;9(3):167-84. doi: 10.1002/oti.163.

28. Law M, Maptiste S, Carswell A, McColl MA, Polatajko HL, Pollock N. Medida Canadense de Desempenho Ocupacional (COPM). Trad. Lívia de Castro Magalhães, Lilian Vieira Magalhães e Ana Amélia Cardoso. Belo Horizonte: Editora Universidade Federal de Minas Gerais; 2009.

29. Kobau R, Dilorio CA, Anderson LA, Price PH. Further validation and reliability testing of the Attitudes and Beliefs about Living with Epilepsy (ABLE) components of the CDC Epilepsy Program Instrument on Stigma. Epilepsy Behav. 2006;8(3):552-9. doi: 10.1016/j.yebeh.2006.01.008.

30. Sachedo RC, Gates JR, Bazil CW, Barkley GL, Tatum W, D Souza J, D’Souza J, McCague K. Improved of quality of life in patients with partial seizures after conversion to oxcarbazepine monotherapy. Epilepsy Behav. 2006;9:457-
63. doi: 10.1016/j.yebeh.2006.04.021

31. Kristensen J, Vestergaard M, Pedersen MG, Pedersen CB, Olsen J, Sidenius P. Incidence and prevalence of epilepsy Denmark. Epilepsy Res. 2007;76(1):60-5. doi: 10.1016/j. eplepsyres.2007.06.012.

32. Frayman L, Cukiert A, Forster C, Ferreira VB, Buratini JA. Qualidade de vida de pacientes submetidos a cirurgia de epilepsia. Arq Neuro-Psiquiatria. 1999;57(1):30-3. doi: 10.1590/S0004-282X1999000100006.

33. Pennell PB, Thompson P. Gender-specific psychosocial impact of living with epilepsy. Epilepsy Behav. 2009; 15(2suppl 1):S20-S25. DOI: 10.1016/j.yebeh.2009.03.022

34. Andrade-Valença LPA, Valença MM, Velasco TR. Epilepsia de lobo temporal mesial associada à esclerose hipocampal. J Epilepsy Clin Neurophsiol. 2006;12(1):31-6. doi: 10.1590/ S1676-26492006000100007.

35. Ferrari CMM. Adesão ao tratamento e qualidade de vida de indivíduos com epilepsia em tratamento ambulatorial [tese]. São Paulo: Escola de Enfermagem da Universidade de São Paulo; 2011.

36. Guerreiro CAM. História do surgimento e desenvolvimento das drogas antiepilépticas. J Epilepsy Clin Neurophysiol. 2006;12(1supp11):18-21. doi: 10.1590/S167626492006000200004.

37. Dilorio C, Yeager K, Shafer PO, Letz R, Henry TR, Schomer DL, McCarty F. The epilepsy medication and treatment complexity index: reliability and validity testing. J Neurosci Nurs. 2003;35(3):155-62. doi: 10.1097/01376517200306000-00005

38. Yeager KA, Dilorio C, Shafer PO, Mc Carty F, Letz R, Henry TR, Schomer DL. The complexity of treatments for persons with epilepsy. Epilepsy Behav. 2005;7:679-86. doi: 10.1016/j.yebeh.2005.07.008.

39. Dilorio C, Shafer PO, Letz R, Henry TR, Schomer DL, Yeager K. Project EASE: a study to test a psychosocial model of epilepsy medication management. Epilepsy Behav. 2004;3:926-36. doi: 10.1016/j.yebeh.2004.08.011.

40. Hovinga CA, Asato MR, Manjunath R, Wheless JW, Phelps SJ, Sheth RD, Pina-Garza JE, Zingaro WM, Haskins LS. Association of non-adherence to antiepileptic drugs and seizures, quality of life, and productivity: survey of patients with epilepsy and physicians. Epilepsy Behav. 2008;13:31622. doi: 10.1016/j.yebeh.2008.03.009.

41. Bastos MC, Lann FV, Gitaí LLG, Gameleira FT, Silva LHBR. Epilepsia MioclônicaJuvenil: Estudo Clínico, epidemiológico, terapêutico e de qualidade de vida. J Epilepsy Clin Neurophsiol. 2009;15(2):65-9. doi: 10.1590/ S1676-26492009000200004.

42. Nickel R, Silvado CE, Germiniani FMB, Paola L, Silveira 
NL, Souza JRB, Robert C, Lima AP, Pinto LM. Quality of life issues and occupational performance of persons with epilepsy. Arq Neuro-Psiquiatria. 1999;70(2):140-4. doi: 10.1590/S0004-282X2012000200013.

43. Anderson KL, Burckhardt CS. Conceptualization and measurement of quality of life as an outcome variable for health care intervention and research. J Adv Nursing. 1999;29(2):298-306. doi: 10.1046/j.13652648.1999.00889.x

44. Dourado MV, Alonso NB, Martins HH, Oliveira ARC, Vancini RL, Lima C, Dubas JP, Cabloco LOSF, Guilhoto LMFF, Yacubian EMT. Quality of life and the selfperception impact of epilepsy in three different epilepsy types. J Epilepsy Clin Neurophysiol. 2007;13(4):191-6. doi: 10.1590/S1676-26492007000400009.

45. Baker GA, Jacoby A, Buck D. Quality of life of people with epilepsy: a European study. Epilepsia. 1997;38:3537-62. doi: 10.1111/j.1528-1157.1997.tb01128.x.

46. Nickel R, Souza JRB, Silveira NL, Robert C. Lima
AP, Navarro EJ, Pinto LM. Estudo descritivo sobre o desempenho ocupacional do sujeito com epilepsia: o uso da CIF como ferramenta para classificação da atividade e participação. Acta Fisiatrica. 2001;18(2):55-9. Disponível em: http://www.actafisiatrica.org.br/detalhe_artigo. asp?id $=78$.

47. Fernandes PT, LI LM. Percepção de estigma na epilepsia. J Epilepsy Clin Neurophysiol. 2006;12(4):207-18. doi: 10.1590/S1676-26492006000700005.

48. Jacoby A. Stigma, epilepsy, and quality of life. Epilepsy Behav. 2002;3:10-20. doi: 10.1016/S1525-5050(02)005450 .

49. Mclin WM, Boer HM. Public perceptions about epilepsy. Epilepsia. 1995;36: 957-9. doi: 10.1111/j.1528-1157.1995. tb00952.x.

50. Jacoby A, Gorry J, Gamble C, Baker GA. Public knowledge, private grief: a study of public attitudes to epilepsy in the United Kingdom and implications for stigma. Epilepsia. 2004;45:1405-15. doi: 10.1111/j.0013-9580.2004.02904.x 